
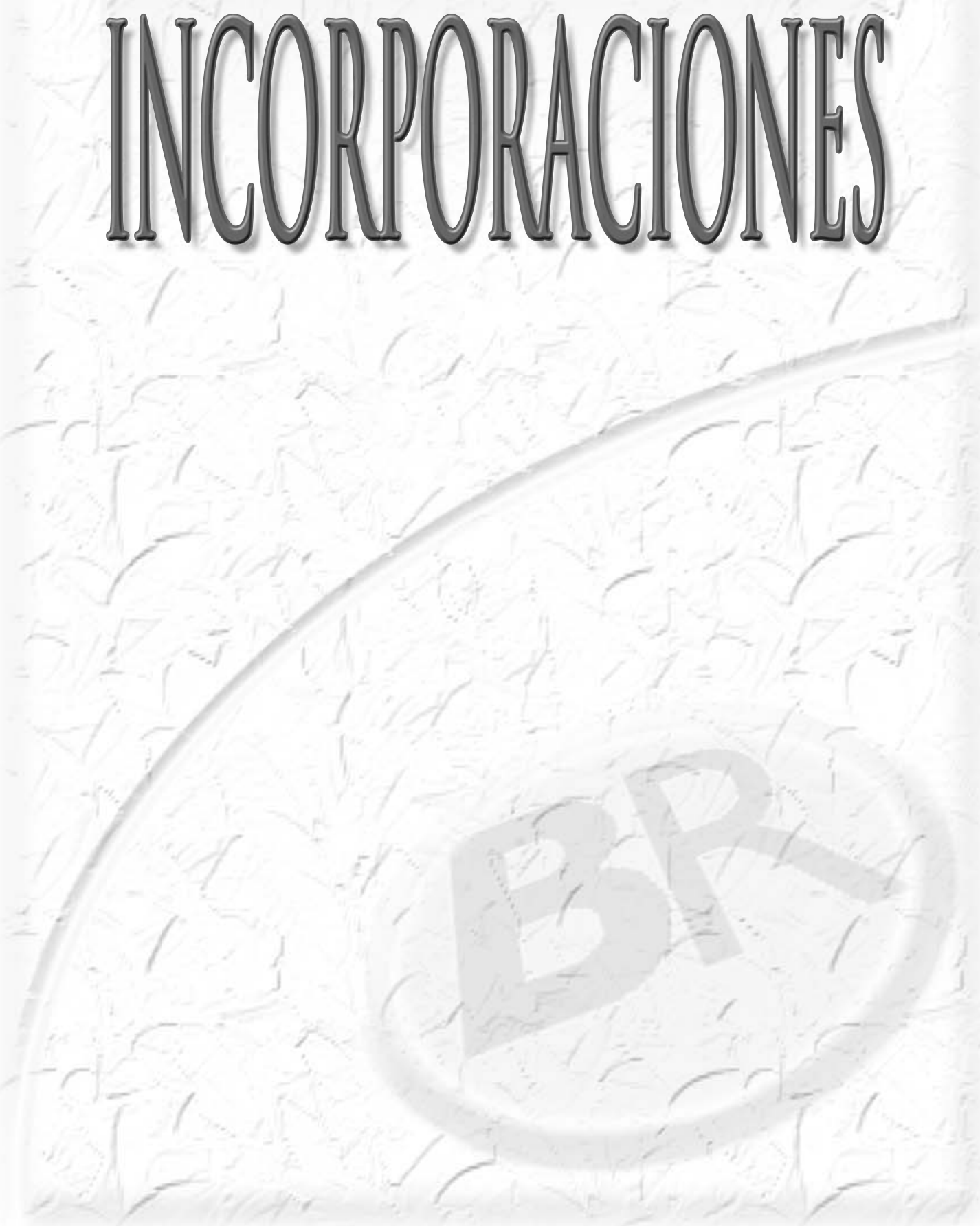



\section{INCORPORACIONES I}

\section{LA AMPLITUD Y DURACIÓN DE LOS EPSPS REGULA LA VENTANA TEMPORAL DEL FENÓMENO DE STDP (SPIKE TIMING DEPENDENT PLASTICITY) (Changes Of The Epsp Waveform Regulate The Temporal Window For Spike Timing Dependent Plasticity).}

Fuenzalida, M.1, ${ }^{2}$, Fernández de Sevilla, D. ${ }^{1}$ y Buño, W. ${ }^{1}$

Instituto Cajal, Consejo Superior de Investigaciones Científicas, Madrid, España. Centro de Neurobiología y Plasticidad del Desarrollo, Departamento de Fisiología, Facultad de Ciencias Universidad de Valparaíso.

Se ha demostrado que la asociación entre la actividad pre y postsináptica puede inducir potenciación o depresión a largo plazo (LTP o LTD), dependiendo del orden temporal entre los potenciales de acción pre y postsinápticos, fenómeno denominado "Spike Timing Dependent Plasticity" (STDP). Anteriormente demostramos que la activación de la conductancia de $\mathrm{K}^{+}$lenta dependiente de $\mathrm{Ca}^{2+}$ (sAHP) modifica la forma de onda de los potenciales postsinápticos excitatorios (EPSP). Aquí investigamos la contribución de la duración y amplitud de los EPSPs en la inducción de LTP por protocolos de STDP (STDP-LTP). Utilizando registros intracelulares en neuronas piramidales de CA1 del hipocampo, generamos STDP-LTP, asociando un EPSP con un potencial de acción postsináptico retropropagado (BAP). Hemos observado que la duración de la ventana temporal de coincidencia entre el EPSP-BAP, requerida para inducir LTP, se reduce cuando disminuye la amplitud y la duración del EPSP como resultado de la activación de la sAHP. Este efecto puede ser revertido con la aplicación de ciclotiazida. Nuestros resultados indican que la amplitud y duración de los EPSPs y su regulación por la SAHP modifica la duración de la ventana temporal para STDP, determinando la polaridad y magnitud de la potenciación sináptica en circuitos neuronales del sistema nervioso central.

Fuentes de Financiamiento: Dirección General de Investigación Científica y Tecnológica, Ministerio de Ciencia y Tecnología (BFI2002-01107 y BFU2005-07486) y Comunidad Autónoma de Madrid (GR/SAL/0877/2004) (W. Buño, España).

\section{C-FOS Y C-JUN PARTICIPAN EN EL COMPLEJO TRANSCRIPTIVO QUE INDUCE LOS GENES PROTECTORES DEL ESTRÉS HIPERTÓNICO.}

Irarrázabal, C., Villanueva, S., Burg M. y Ferraris, J. Universidad de los Andes

Las células del riñón retienen agua y electrolitos. La médula renal es expuesta a estrés hipertónico alcanzando hasta $4000 \mathrm{mOsm}$ comparado con $300 \mathrm{mOsm}$ del plasma. El factor de transcripción TonEBP es inducido y activado por hipertonicidad. TonEBP induce la expresión de genes osmoprotectores, tales como aldosa reductasa (AR) y el transportador de botaina/acido y amino butírico (BGT1). La pérdida de TonEBP origina atrofia renal y pérdida de los factores osmoprotectores. En la región promotora de estos genes existen las secuencias de respuesta a osmolaridad (ORE) y AP-1 (familia Fos y Jun). En la presente investigación describimos la participación de cFos y c-Jun en la actividad transcriptiva inducida por hiperosmolaridad. Utilizando un sistema de gen reportero con el promotor de AR (ORE:AP-1), se pudo establecer que AP-1 participa en la transcripción inducida por hipertonicidad. El mismo resultado se obtuvo con un dominante negativo de AP-1. La disminución de la expresión de c-Fos y c-Jun (siRNA) reduce el mRNA de AR y BGT1. La inhibición de p38 (activador de TonEBP) reduce la actividad del gen reportero, dependiente de AP-1. Utilizando sondas de ADN (ORE:AP1) se estableció que TonEBP, c-Fos y c-Jun se asocian en un mismo complejo a la secuencia ORE:AP1. Además, c-Fos y c-Jun coinmunoprecipitan con TonEBP, sugiriendo una asociación física. Estos datos permiten sugerir la participación de c-Fos y c-Jun en el complejo transcriptivo activado por hipertonicidad.

\section{PARCICIPACIÓN DE PROTEÍNA QUINASA C EN LA ACTIVACIÓN DE NADP(H) OXIDASA POR INSULINA EN MIOTUBOS (Protein kinase $\mathrm{C}$ role in $\mathrm{NADP}(\mathrm{H})$ oxidase activation induced by insulin in myotubes)}

Espinosa, A., Hidalgo, C. y Jaimovich, E.

Centro de Estudios Moleculares de la Célula, Facultad de Medicina, Universidad de Chile.

NADPH oxidasa es una enzima que cataliza la producción de ión superóxido, es un complejo compuesto por: p40 phox, p47 phox ,p67pho, $\mathrm{p} 22^{\text {phox }}$ y gp91 ${ }^{\text {phox }}$; subunidades citosólicas y $\mathrm{p} 22^{\text {phox }}$ y gp91 phox , localizadas en membranas formando el complejo b558. Cuando p47phox es fosforilado, migra hacia membranas, uniéndose al complejo b558, activando la enzima. Se ha descrito que insulina produce especies reactivas del oxígeno dependiente de NADPH en adipocitos humanos. En este trabajo caracterizamos la presencia de gp91 phox y p47phox en miotubos de músculo esquelético de ratas. Estudiamos la activación de $\mathrm{NADP}(\mathrm{H})$ oxidasa por insulina, utilizando 5-(-6)-chloromethyl-2',7'diclorodihidrofluoresceina diacetato; una sonda fluorescente sensible a $\mathrm{H}_{2} \mathrm{O}_{2}$ y observamos la translocación de p47phox mediante inmunofluorescencia indirecta. Evaluamos la participación de la proteína quinasa C (PKC) utilizando inhibidores específicos. Insulina produce ROS a los 15 min después del estímulo, este efecto es inhibido por apocinina e inhibidores de PKC. p4 $7^{p h o x}$ está presente en los myotubos mostrando un patrón estriado en condiciones basales, insulina produce la translocación de p47phox a estructuras que colocalizan con actina sarcomérica. Este efecto es inhibido por apocinina e inhibidores de PKC. Los resultados indican que $\mathrm{NADP}(\mathrm{H})$ oxidase esta presente en miotubos, es activada por insulina para producir ROS y esta activación es dependiente en parte de PKC.

FONDAP 15010006 


\section{INCORPORACIONES II}

\section{FACTORES ASOCIADOS CON LA INTRODUCCIÓN Y ESTABLECIMIENTO DE LA MACROALGA NO INDÍGENA, CODIUM FRAGILE (CHLOROPHYTA) EN LA COSTA DE CHILE (Factors associated with the introduction and establishment of the non-indigenous macroalga, Codium fragile (Chlorophyta) on the Chilean coast).}

\begin{abstract}
Neill, P.E. ${ }^{1,2}$, Correa, J.A. ${ }^{2}$ y Navarrete, S.A. ${ }^{2,3}$
${ }^{1}$ Departamento de Ecología Costera, Facultad de Ciencias, Universidad Católica de la Santísima Concepción, Concepción. ${ }^{2}$ Center for Advanced Studies in Ecology \& Biodiversity (CASEB) y Departamento de Ecología. Pontificia Universidad Católica de Chile. Santiago. ${ }^{3}$ Estación Costera de Investigaciones Marinas, Pontificia Universidad Católica de Chile, Santiago.
\end{abstract}

La ecología de invasiones intenta entender y predecir patrones de dispersión y éxito de establecimiento de especies no indígenas, como un importante componente de cambio global. En este trabajó se evaluó la extensión geográfica de una reconocida macroalga invasora a lo largo de la costa de Chile, y se utilizó regresión logística para generar un modelo que explica su ocurrencia basado en variables asociadas con dispersión y condiciones ambientales. El alga presenta una distribución discontinua y parchosa, marcada por dos notables agrupaciones de sitios en cada extremo del rango, y sitios solitarios intermedios. Los resultados claramente apoyan actividades de acuicultura exótica y nativa como importantes factores asociados a la introducción y dispersión del alga en la costa chilena. Además, la presencia de C.fragile en un amplio rango de temperatura sugiere que no existe una barrera térmica a su establecimiento. Proponemos dos posibles escenarios de introducción y expansión de esta macroalga en Chile.

CONICYT(AT-4040050), IdeaWild, PEO Scholar Award.

\section{ADAPTIVE RADIATION IN EVOLUTION OF ECOMORPHOLOGICAL TRAITS INSIDE ABROTHRICHINI (RODENTIA: SIGMODONTINAE) Radiación Adaptativa en la Evolución de Rasgos Ecomorfológicos de Abrothrichini (Rodentia: Sigmodontinae).}

Rodríguez-Serrano, E. ${ }^{\mathrm{a}}{ }^{*}$, Hernández, C.E. ${ }^{\mathrm{a}, \mathrm{b}}$ \& Palma, R.E. , $^{\mathrm{b}}$

a Laboratorio de Biología Evolutiva, Departamento de Ecología, Facultad de Ciencias Biológicas, and Center for Advanced Studies in Ecology \& Biodiversity, Pontificia Universidad Católica de Chile, Chile. ${ }^{\text {b }}$ Laboratorio de Diversidad Molecular y Filoinformática, Departamento de Zoología, Universidad de Concepción, Concepción, Chile.

The generally accepted hypothesis regarding the origin of fossorial mammals proposes adaptive convergence from open environments towards the use of subterranean environments. We evaluated this hypothesis for South American mole-mice using a multiple approach with independent evidence. Utilizing a molecular approached based on Cytochrome b and IRBP sequences, we evaluated phylogenetic relationships, time of origin, the ancestral trait of fossoriality and ancestral distributions of species belonging to the Andean Clade (Rodentia; Sigmodontinae). Our results indicate that the Andean Clade is highly sustained; with one clade grouping all fossorial species and another grouping all cursorial species. Fossoriality originated in the Middle-Pliocene in Temperate Forests of southern South America from a widely distributed cursorial ancestor inhabiting closed environments. We conclude that the origin of fossorial ecomorphological traits did not necessarily occur under a general model of open environments, rather the origin of these traits depends on the environment in which these species originated, as well as their ecological-historical relationship with the environment.

Acknowledgements: DIUC-Patagonia 205.113.0651sp; FONDAP-FONDECYT grant 1501-0001 Program 2 CASEB; CONICYT Doctoral fellowship. 